Die Erfahrung lehrt, daß man ungeschützte Drosselspulen viele Male ausschalten kann, ohne daß sie durchschlagen. Unsere Fig. 4 gibt die Erklärung. Erfolgt die Abschaltung ,genügend langsam“, so schlägt die Spule nicht durch. „Genügend langsam" heißt in diesem Falle, die Ausschaltzeit muß größer sein als die Schwingungszeit aus Spule und einen ihr parallel geschalteten nach 3) bemessenen Kondensator. Sobald die Abschaltung rasch genug erfolgt, schlägt die Spule durch.

Beim Abschalten einer Induktivität wird man daher Schalter, die eine langsame Unterbrechung begünstigen; vorziehen. Schnappschalter sind, wie die Praxis bereits längst herausgefunden hat, ganz zu verwerfen. Es wäre lohnend, für eine Reihe gebräuchlicher Schalter durch den Versuch Ausschaltkurven aufzunehmen und ihre Spannungen mit der vorgetragenen Theorie zu vergleichen.

\title{
Grundlagen zur Konstruktion eines neuen Durchführungsisolators.
}

\author{
Von
}

Adolf Bolliger, Zürich.

Im 8. und 9. Hefte, Jahrg. I9I4 dieser Zeitschr., erschien die Arbeit über Hochspannungsisolatoren von Karl Kuhlmann, zu deren Mitarbeit ich herangezogen worden war. Damals faßte ich die Ideen, welche mich kurz darauf zur Konstruktion des in dieser Schrift besprochenen Durchführungsisolators geführt haben. Die Konstruktion von Kraftlinienbildern läßt leicht die unzweckmäßige Verteilung des Feldes bei den bestehenden Durchführungsisolatoren erkennen, ist aber auch imstande, die der günstigsten Potentialverteilung entsprechenden Leiterformen zu finden. Daß hierfür den

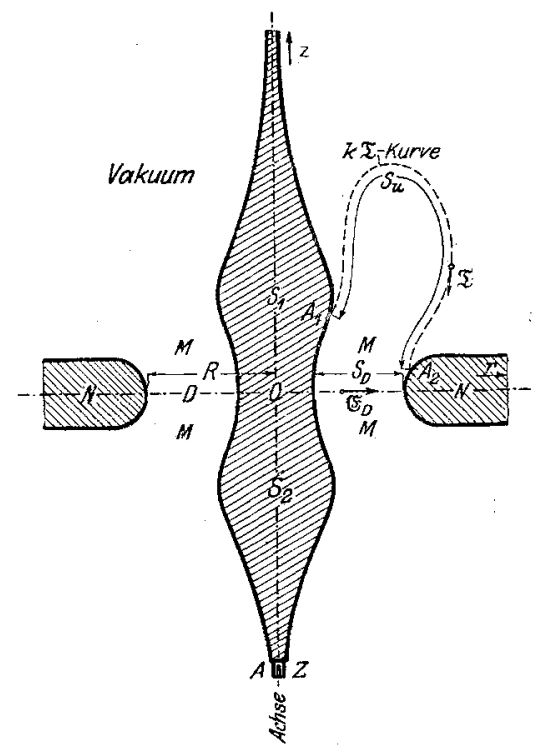

Fig. 1 . graphischen Methoden gegenüber den analytischen Methoden der Vorzug zu geben ist, dürfte zum Beispiel der Vergleich von Fig. I vorliegender Arbeit mit Fig. 3 der seitdem von J. Spielrein erschienenen Abhandlung (3. Heft, Jahrg. I9I5 dieser Zeitschr.) zeigen. Spielrein hat dort versucht, eine Luftdurchführung mit möglichst konstanter Feldstärke zu konstruieren. Er hat für das Verhältnis der Radien an der weitesten und engsten Stelle seines Hochspannungsbolzens, welches für die günstigsten Leiterformen eine Konfigurationskonstante darstellt, den Wert 4 erhalten, während meine Fig. I und 2 den Wert 1,6 ergeben. Die entsprechende Abänderung meines Kraftlinienbildes (Fig. 2) auf die von Spielrein ermittelten Leiterformen würde zeigen, daß bei jener Durchführung am Wulste des Hochspannungsbolzens und am innern Rande der Niederspannungsfassung relativ hohe Feldstärken auftreten würden. Um die richtige Potentialverteilung im Innern des Isolators zu erreichen, muß man auch das Verhalten des Feldes in der Umgebung desselben betrachten. Das Kraft- bzw. D-Linienbild dieser Schrift ist nach dem in der Kuhlmannschen Arbeit angegebenen Verfahren gezeichnet worden und entspricht - wie dies übrigens auch bei den dortigen Bildern der Fall ist - rotationsförmigen Leitern. Spielrein hat dies in seiner Arbeit 
für die $\mathrm{Kuhlmannschen} \mathrm{Bilder} \mathrm{bestritten} \mathrm{und} \mathrm{dieselben} \mathrm{als} \mathrm{sogenannte} \mathrm{„ebene“} \mathrm{Bilder}$ ausgegeben. Es läßt sich aber leicht einsehen, daß dem nicht so ist; denn für ebene Bilder ist bekanntlich das Verhältnis der Länge $\mathrm{ds}$ zur Breite $\mathrm{db}$ für alle Vierecke konstant, wenn man nur Niveaulinien mit gleichen Potentialunterschieden und Flußröhren mit gleichen Verschiebungsmengen betrachtet. Der Beweis dafür ist übrigens sehr leicht zu erbringen. Die zweite Gleichung auf Seite 209 von Kuhlmanns Arbeit und dessen Kraftlinienbilder belehren uns aber vom Gegenteil, Bei näherer Betrachtung erkennt man, daB das Verhältnis $\lambda=\frac{\mathrm{ds}}{\mathrm{db}}$ der Viereckseiten proportional dem $\mathrm{Ab}$ stand $\mathrm{r}$ von der Bolzenachse ist, wie dies bei Bildern in den Meridianebenen von rotationsförmigen Feldern der Fall ist.

\section{Konstruktionsprinzipien für den Isolator.}

Für die Konstruktion eines guten Isolators sind die folgenden drei Einzelaufgaben zu lösen:

I. Bestimmung zweckmäßiger Leiterformen.

II. Bestimmung der Form des Isolatorkörpers unter Berücksichtigung eines geeigneten Isolationsmaterials.

III. Sicherung des Isolators gegen betriebsstörende, äußere Einflüsse.

Zu Aufgabe I. Um den Begriff: „Was sind zweckmäßige Leiterformen“ fassen zu können, denken wir uns zwischen der Hochspannungsleitung und der Niederspannungsfassung den leeren Raum. Wir . sehen also vollständig ab von der höheren Dielektrizitätskonstanten des Isolatorkörpers. Die Leiterformen nehmen wir in bezug auf die Durchführungsstelle D (vgl. Fig. I) symmetrisch an. Bezeichnen dann $E_{\max }$ und $\aleph_{\min }$ den maximalen und den minimalen Wert von $\mathbb{E}_{\mathrm{D}}$ an der Durchführungsstelle $\mathrm{D}$, so definieren wir solche Leiter als zweckmäßig, für welche die Größe

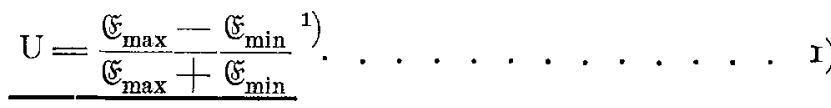

für eine bestimmte Potentialdifferenz der Leiter $\mathrm{zu}$ einem Minimum wird. $\mathrm{U}$ soll Ungleichförmigkeitsgrad der Feldstärke $\mathfrak{E}_{D}$ heißen.

Je kleiner $U$ ist, um so mehr nähern wir uns dem Idealfalle, wo die Feldstärke $\mathbb{E}_{D}$ in der ganzen Durchführungsstelle gleich groß ist. Es bedarf wohl keiner nähern Begründung, warum die vollständige Erreichung dieses Zieles unmöglich ist; hingegen ist leicht einzusehen, daß es für $U$ einen mathematischen Minimalwert gibt.

Leider steht $U$ mit den Leiterformen in so komplizierter Abhängigkeit, daß die praktische Ausführung des hier vorliegenden Variationsproblemes rein ausgeschlossen ist. Für die Ermittlung der günstigsten Leiterformen bleibt schließlich nur der Weg des Probierens, den der Verfasser auch eingeschlagen hat. Das Probieren besteht hier darin, daß zu angenommenen Leiterformen jeweilen das ganze Kraftlinienbild konstruiert wird und unter möglichst vielen Fällen der günstigste Fall herausgegriffen wird. Der Verfasser hat so die Leiterformen, wie sie die Fig. I und 2 zeigen, gefunden.

Fig. 2 enthält das zu den ermittelten Leiterformen gehörende Vakuumfeld. Man erkennt daran, daß die Niveaulinien in der Gegend der Durchführungsstelle $D$ nahezu äquidistant sind; das heißt, daß die Feldstärke $\&$ im Mittelteile $\mathrm{M}$ des Isolators praktisch konstant ist.

1) Wenn man ganz genau verfahren wollte, so müften in der Bedingungsgleichung für die giinstigsten Leiterformen auch die Feldstärken auferhalb der Durchführungsstelle $D$ in Betracht gezogen werden. An Stelle der Gleichung I) würde ein Gesetz treten, das die weitere Untersuchung wesentlich erschweren und ein nur wenig verändertes Resultat ergeben würde. 
Der Hochspannungsleiter $\mathrm{L}_{1}$ ist zu einer eingeschnürten Doppelspindel ausgebildet, und die Niederspannungsfassung ist scheibenförmig und wird gegen innen ungefähr kreisringförmig begrenzt. Die Feldstärke erreicht ihren Maximalwert nicht an der Durchführungsstelle D, sondern - was besser ist — am äußern Rande der beiden Spindelköpfe $\mathrm{S}_{1}$ und $\mathrm{S}_{2}$; denn der Weg der dort beginnenden Kraftlinien ist länger als der Kraftlinienweg in der Durchführungsstelle D.

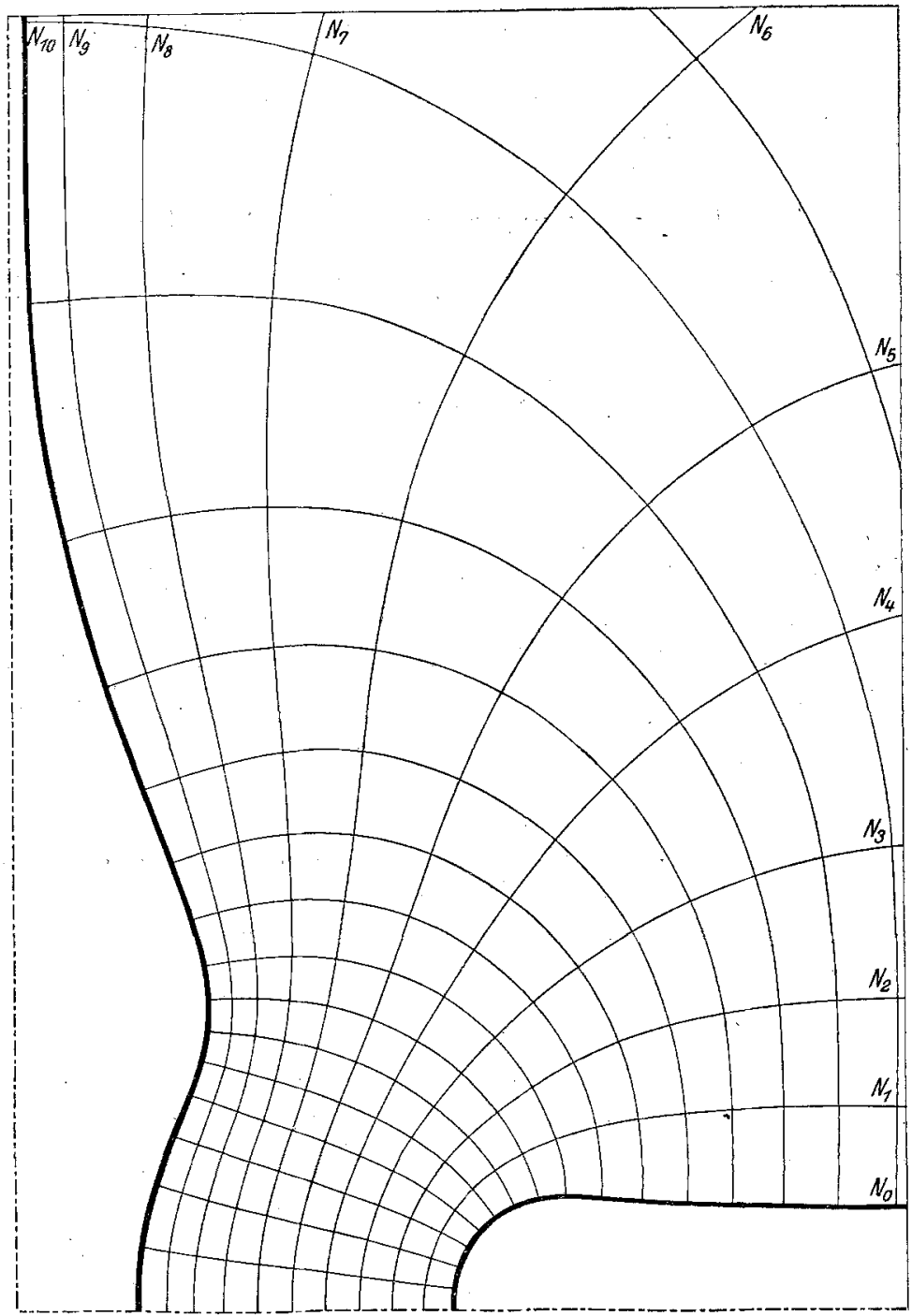

Fig. 2. Meridianschnitt durch das Vakuumfeld.

$Z_{u}$ Aufgabe II. Für die Sicherheit des Isolators gegen Überschlag ist eine längs der Oberfläche des Isolatorkörpers überall gleichbleibende Tangentialfeldstärke das günstigste, weil dann jeder Punkt der Oberfläche mit der zulässigen Úberschlagsfestigkeit beansprucht werden kann. Um einen Isolatorkörper zu konstruieren, der dieser Bedingung näherungsweise entspricht, betrachten wir im nächsten Abschnitte Kurven konstanter Tangentiaifeldstärke. 


\title{
Kurven konstanter Tangentialfeldstärke.
}

\author{
$(\mathrm{k} \cdot \mathfrak{T}$-Kurven.
}

In irgendeiner Fläche (K-Fłäche), die von einer Kraft- bzw. D-Linie nirgends geschnitten wird, also aus lauter Kraft- bzw. D-Linien besteht, kann man Kurven konstanter Tangentialfeldstärke ( $\mathrm{k} \cdot \mathfrak{T}$-Kurven) betrachten. Eine solche Fläche bilden z. B. alle Kraft- bzw. D-Linien, welche durch irgendeine Niveaulinie des Feldes gehen.

Die Ermittlung der $\mathrm{k} \cdot \mathfrak{T}$-Kurven ist unter der Voraussetzung möglich, daß die Potentialverteilung des Feldes längs der ganzen K-Fläche bekannt ist.

Für die analytische Untersuchung beschränken wir uns auf den Fall ebener Kurven. Wir nehmen also an, daß das vorliegende Feld einen oder mehrere ebene Schnitte senkrecht zu allen getroffenen Niveaulinien zuläßt und bestimmen dann den Verlauf der $\mathrm{k} \cdot \mathfrak{T}$-Kurven in derartigen Schnittflächen. Für die sog., ,ebenen Feldverteilungen", denen das sog. Logarithmische Potential, das nur von zwei zu der Ebene parallelen Koordinaten abhängig ist, zugrunde liegt, wird deshalb z. B. die Aufgabe vollständig gelöst.

Wir beziehen in unserer K-Ebene alles auf rechtwinklige, geradlinige Koordinaten ( $\mathrm{x}, \mathrm{y})$. Bezeichnet $\varphi(\mathrm{x}, \mathrm{y})$ oder kürzer $\varphi$ das Potential längs dieser Ebene und $\Phi$ einen

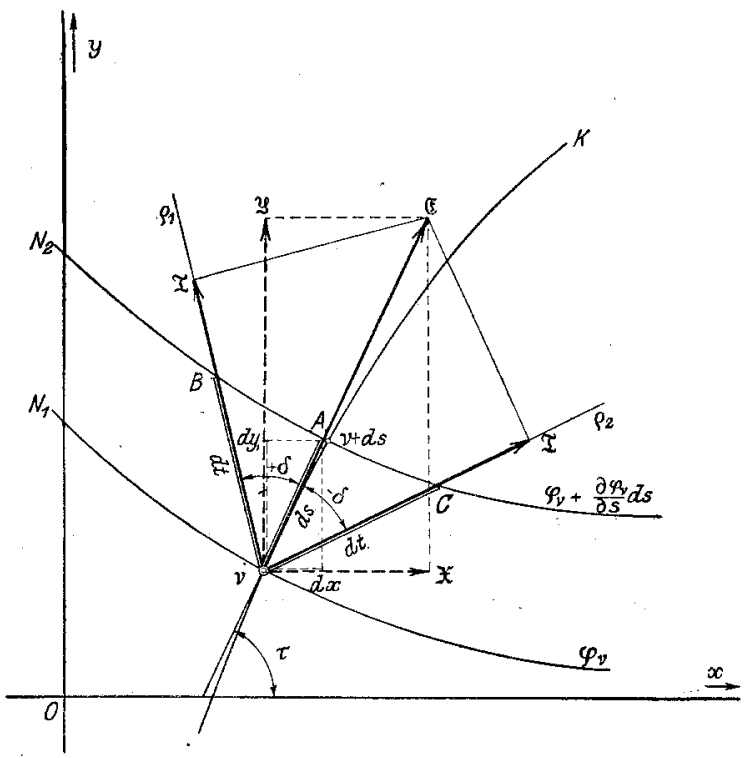

Fig. 3. konstanten Parameter, so kann die Schar der Niveaulinien in der K-Ebene durch die folgende Gleichung dargestellt werden:

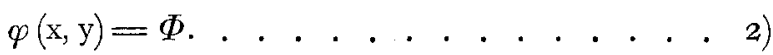

Die Kraftlinien bilden dazu die Schar der orthogonalen Trajektorien.

Bedeuten in Fig. $3 \mathfrak{X}$ und $\mathfrak{Y}$ die $x$ - und y-Komponenten der Feldstärke $\mathbb{E}$ und $\mathrm{dx}$ und $\mathrm{dy}$ die $\mathrm{x}$ - und $\mathrm{y}$-Komponenten des Linienelementes ds der Kraftlinie $\mathrm{K}$ im Punkte $y$, so gilt nach der Abbildung die Beziehung:

$$
\frac{\mathrm{dy}}{\mathrm{d} \mathrm{x}}=\frac{\mathfrak{Y}}{\mathfrak{x}}=\frac{\frac{\partial \varphi}{\partial \mathrm{y}}}{\frac{\partial \varphi}{\partial \mathrm{x}}} \text {. . . . . . . . . . . . . . . 3 }
$$

und dies ist die Differentialgleichung der Kraftlinien.

In jedem Punkte $\boldsymbol{y}$ gibt es zwei Richtungen $\varrho_{1}$ und $\varrho_{2}$, die mit der Kraftlinie K entgegengesetzt gleiche Winkel $+\delta$ und $-\delta$ einschließen, für welche die Komponenten der Feldstärke $\mathfrak{E}$ die Größe $\mathfrak{I}$ haben, so lange

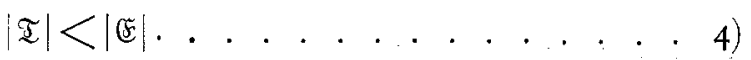

Um die Differentialgleichung der $k \cdot \mathfrak{T}-K u r v e n$ ableiten zu können, muß berücksichtigt werden, daß in der Differentialgleichung 3) der Kraftlinien

$$
\frac{\mathrm{dy}}{\mathrm{dx}}=\mathrm{y}^{\prime}=\operatorname{tg} \tau
$$


ist, und man erhält deshalb die Differentialgleichung der $\mathrm{k} \cdot \mathfrak{I}$-Kurven, wenn man $\tau$ durch $\bar{\tau}=\tau \pm \delta$ ersetzt. Es ist dann:

$$
\left.\operatorname{tg} \bar{\tau}=\operatorname{tg}(\tau \pm \delta)=\frac{\operatorname{tg} \tau \pm \operatorname{tg} \delta}{\mathrm{I} \overline{+} \operatorname{tg} \tau \operatorname{tg} \delta} . \ldots . . . . .6\right)
$$

Um $\operatorname{tg} \delta$ berechnen zu können, wenden wir das Gesetz.

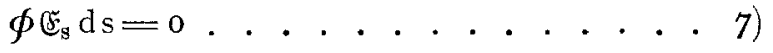

auf das Dreieck $\mathrm{AB} v$ der Fig. (3) an. Es folgt dann, wenn ds den Normalabstand und $\mathrm{dt}$ die Länge der Strecken in den Richtungen $\varrho_{1}$ und $\varrho_{2}$ zwischen den Niveaulinien $\mathrm{N}_{1}$ und $\mathrm{N}_{2}$ an der Stelle $v$ bedeuten:

$$
\cos \delta=\frac{\mathrm{ds}}{\mathrm{dt}}=\frac{\mathfrak{T}}{\mathfrak{E}}, . . . . . . . . .8
$$

woraus:

$$
\left.\operatorname{tg} \delta=\frac{\sqrt{\mathfrak{E}^{2}-\mathfrak{I}^{2}}}{\mathfrak{I}} . . . . . . . . . . .99\right)
$$

Unter Berücksichtigung von 3), 5) und 9) folgt aus 6) die Differentialgleichung der Kurven konstanter Tangentialfeldstärke zu:

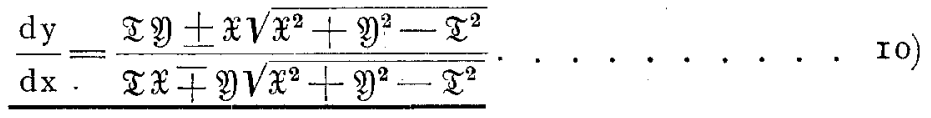

Hierin sind $\mathfrak{I}$ die konstant anzunehmende Tangentialfeldstärke und $\mathfrak{X}$ und $\mathfrak{V}$ die $\mathrm{x}$ - und y-Komponenten der elektrischen Feldstärke $\mathbb{E}$, die man aus dem Potentiale $\varphi$ durch die folgenden Differentiationen nach den Koordinatenrichtungen erhält:

$$
\left.\left.\begin{array}{l}
\mathfrak{X}=-\frac{\partial \varphi(\mathrm{x}, \mathrm{y})}{\partial \mathrm{x}} \\
\mathfrak{V}=-\frac{\partial \varphi(\mathrm{x}, \mathrm{y})}{\partial \mathrm{y}}
\end{array}\right\} \cdot . \cdot \cdot . . . . . \mathrm{II}\right)
$$

Durch Integration der Gl. ro) erhält man, je nachdem man dort die obern oder untern Vorzeichen verwendet, die eine oder andere der beiden $\mathrm{k} \cdot \mathfrak{T}$-Kurvenscharen. Die Größe $\mathfrak{I}$ übernimmt dabei die Rolle des Kurvenparameters.

Anwendungsbeispiel. $\mathrm{k} \cdot \mathfrak{T}-\mathrm{Kurven}$ in einer Meridianebene des Feldes eines unendlich langen, geradlinigen, linearen Leiters. Wir legen den geradlinigen Drahtleiter in die y-Achse unseres rechtwinkligen Koordinatensystems ( $x, y)$. Dann ist:

$$
\left.\varphi=\mathrm{c} \lg \frac{\mathrm{I}}{\mathrm{x}} \text {. . . . . . . . . . . . . . . I } 2\right)
$$

und nach II):

Also lautet für diesen Fall 'Gl. Io):

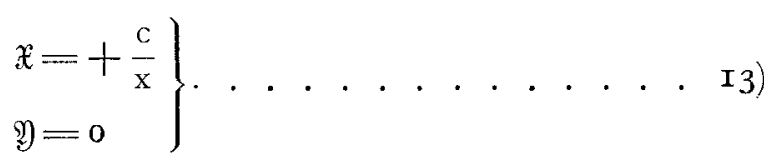

$$
\left.\frac{\mathrm{dy}}{\mathrm{dx}}= \pm \frac{\sqrt{\mathfrak{X}^{2}-\mathfrak{I}^{2}}}{\mathfrak{I}}= \pm \frac{\sqrt{\mathrm{c}^{2}-(\overline{\mathfrak{I} x})^{2}}}{\mathfrak{I x}}, \ldots . . . . . \mathrm{I} 4\right)
$$

woraus durch Separation der Variabeln und Integration folgt:

$$
y= \pm \frac{c}{\mathfrak{T}} \int \frac{\sqrt{\mathrm{x}-\left(\frac{\mathfrak{T} x)^{2}}{c}\right.}}{x} \mathrm{dx}+\mathrm{C}_{1}
$$


Durch die Konstante $C_{1}$ wird offenbar nur der Anfangspunkt des Koordinatensystems festgelegt; da sich aber das Potential $\varphi[$ Gl. I2)] in der y-Richtung überhaupt nicht ändert, kann man mit Vorteil

setzen.

$$
\mathrm{C}_{1}=0
$$

Nach Ausführung der Integration in Gl. I5) ergibt sich für y das Resultat:

$$
\left.\mathrm{y}= \pm \frac{\mathrm{c}}{\mathfrak{I}}\left\{\frac{\sqrt{\mathrm{c}^{2}-(\mathfrak{I x})^{2}}}{c}+\lg \mathrm{n} \frac{\mathrm{c}-\sqrt{\mathrm{c}^{2}-(\mathfrak{I x})^{2}}}{\mathfrak{I} \mathrm{x}}\right\} . . . \mathrm{I} 6\right)
$$

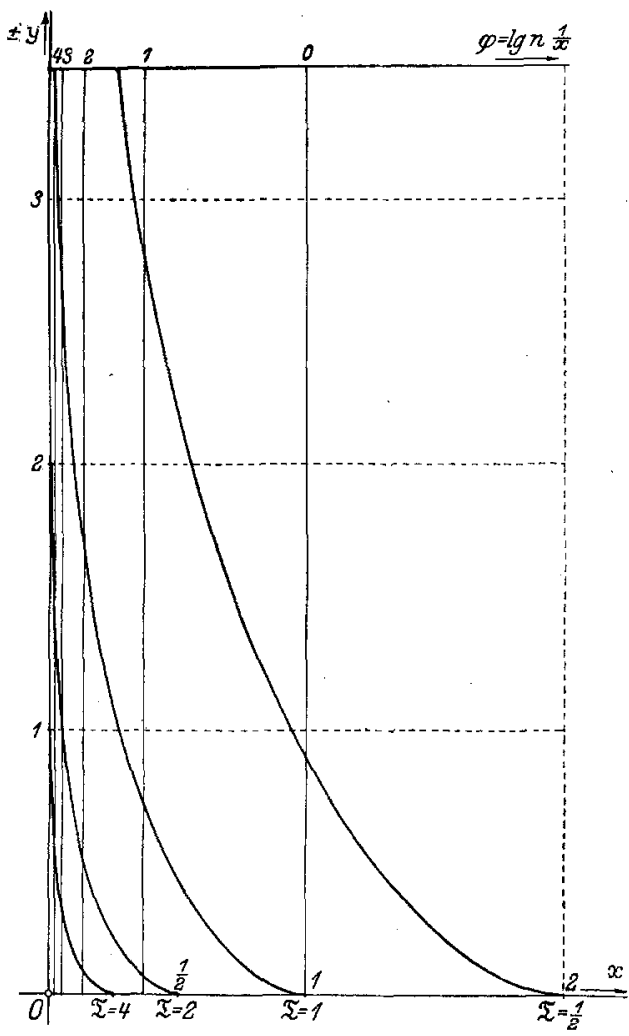

Fig. 4.

Das Resultat Gl. I6) wird in Fig. 4 graphisch veranschaulicht. Man erkennt sowohl aus der Formel, wie auch aus der Abbildung, $\mathrm{da} B$ für $\mathrm{x}=\mathrm{o}$ alle $\mathrm{k} \cdot \mathfrak{T}$-Kurven eine Singularität besitzen, und zwar werden dort alle Werte von y logarithmisch unendlich. Die Leiterachse ist Asymptote für die Kurven. Der Grund liegt darin, daß am Leiter das Potential von unendlich großen Werten auf endliche Beträge fällt. Um diese Potentialdifferenz mit der endlichen Feldstärke $\mathfrak{I}$ zu leisten, muß längs des Leiters ein unendlich großer Weg durchlaufen werden.

Für

$$
\left.\mathrm{x}=\frac{\mathrm{c}}{\mathfrak{I}} \cdot \ldots \cdot \cdot \mathrm{x} 7\right)
$$

enden die Kurven. Darüber hinaus ist nämlich die Feldstärke \& kleiner als die vorgeschriebene Tangentialfeldstärke $\mathfrak{I}, d$. h. die Werte y der Gl. I6) werden imaginär. In den Endpunkten der Kurven ist $\mathbb{E}=\mathfrak{I}$, und die Kurventangenten stehen senkrecht auf den $\mathrm{Ni}$ veaulinien ${ }^{\mathbf{1}}$ ).

Aus dem Besprochenen und aus der Fig. 4 gehen deutlich die Eigenschaften hervor, welche den $\mathrm{k} \cdot \mathfrak{T}-K u r v e n$ im allgemeinen zukommen, und deren Verständnis für die Anwendung auf die Isolatorenkonstruktion nötig ist.

Graphische Näherungsmethode. Ist die Potentialverteilung nicht durch ein analytisches Gesetz, sondern durch die Angabe möglichst vieler Niveaulinien mit der konstanten Spannungsdifferenz $\Delta \varphi$ festgelegt, so kann der Verlauf der $\mathrm{k} \cdot \mathfrak{T}$-Kurven näherungsweise auf graphischem Wege ermittelt werden. Wir nehmen zu diesem Zwecke das an der Stelle $\boldsymbol{\nu}$ zwischen zwei benachbarten Niveaulinien liegende $\mathrm{k} \cdot \mathfrak{T}$-Kurvenstück von der Länge $\Delta \mathrm{t}$ als geradlinig an. Ist dann $\mathfrak{I}$ die konstant angenommene Tangentialfeldstärke, so muß sein:

$$
\mathfrak{T} \cdot \Delta t=\Delta p
$$

1) Das bei den Isolatoren vorkommende Potential nähert sich in der Umgebung des Hochspannungsbolzens mehr oder weniger dem in diesem Abschnitt besprochenen Potential des linearen Leiters. Die k. I-Kurven müssen deshalb dort einen ähnlichen Verlauf haben wie in Fig. 4. Es erklärt sich hieraus der Vorteil der von der Firma Brown-Boveri \& Cie. eingeführten konkaven Isolatorform gegenüber der früher allgemein ïblichen konvexen Form. 
d. h.

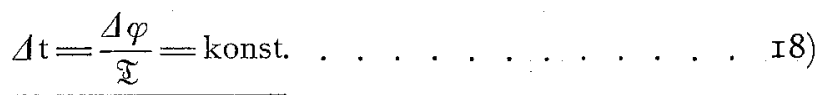

Die nach Gl. I8) berechnete Strecke kann man also leicht zwischen den Niveaulinien mit dem Zirkel in stetiger Aneinanderreihung abtragen. Der ungefähre Verlauf der $\mathrm{k} \cdot \mathfrak{I}$-Kurven ergibt sich dann in Form von Polygonzügen. Die so gewonnenen Kurven werden bei exaktem Konstruieren um so genauer den wahren $\mathrm{k} \cdot \mathfrak{I}$-Kurven entsprechen, je kleiner die Potentialdifferenz $\Delta \varphi$ gewählt wird.

\section{Konstruktion des Isolatorkörpers.}

Die Form des einer konstanten Tangentialfeldstärke entsprechenden Isolatorkörpers läßt sich mit Hilfe der $\mathrm{k} \cdot \mathfrak{T}$-Kurven des leeren Raumes unter der Voraussetzung von relativ dünnen Isolatormänteln näherungsweise finden. Ein durch solche Kurven begrenzter Isolatorkörper besitzt im definitiven Isolatorfelde eine ungefähr konstante Tangentialfeldstärke.

Jeder Durchführungsisolator muß so gebaut sein, daß seine Durchschlagsfestigkeit größer ist als seine Sicherheit für das Auftreten von Gleitfunken. Für einen „Luftisolator", auf dessen genauere Untersuchung wir uns in dieser Schrift beschränken wollen, erweisen sich die folgenden Sicherheitskoeffizienten als zweckmäßig:

$$
\left.\begin{array}{l}
\text { Sicherheitskoeffizient gegen Durchschlag }=3 \\
\text { Sicherheitskoeffizient gegen Überschlag }=2
\end{array}\right\}
$$

Nun besitzt aber die atmosphärische Luft eine Durchschlagsfestigkeit von maximal $30000 \mathrm{~V} / \mathrm{cm}$ und erträgt längs Porzellan eine Tangentialfeldstärke von mindestens $\left.4000 \mathrm{~V} / \mathrm{cm}^{1}\right)$. Daher kann man einen Luftisolator so bauen, daß er entsprechend den Sicherheitskoeffizienten [Gl. I9)] an der Durchführungsstelle D mit einer Feldstärke:

$$
\mathfrak{E}_{\mathrm{D}}=\mathrm{I0} 000 \mathrm{~V} / \mathrm{cm} \text {. . . . . . . . . . . . 20) }
$$

und längs des Porzellanisolators mit der Tangentialfeldstärke

$$
\mathfrak{I}=2000 \mathrm{~V} / \mathrm{cm} \text {. . . . . . . . . . . . . } 2 \mathrm{I})
$$

beansprucht wird.

Bezeichnet $\mathrm{E}$ die ganze Spannungsdifferenz des Isolators, $\mathrm{S}_{\mathrm{D}}$ den totalen Durchschlagsweg (vgl. Fig. I) und $\mathrm{S}_{U}$ den totalen Überschlagsweg, so gilt der Annahmen von konstanten Feldstärken $\mathfrak{E}_{\mathrm{D}}$ und $\mathfrak{T}$ wegen

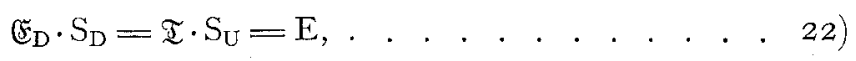

woraus:

$$
\mathrm{S}_{\mathrm{U}}=\mathrm{S}_{\mathrm{D}} \cdot \frac{\mathfrak{E}_{\mathrm{D}}}{\mathfrak{I}}
$$

Wenn nun im Kraftlinienbild ( $\mathrm{n}-\mathrm{I}$ ) Niveaulinien eingezeichnet sind, so folgt für die Strecke $\Delta \mathrm{t}$ der Formel $\mathrm{I} 8$ )

$$
\Delta \mathrm{t}=\frac{\mathrm{S}_{\mathrm{U}}}{\mathrm{n}}=\frac{\mathrm{S}_{\mathrm{D}}}{\mathrm{n}} \cdot \frac{\mathfrak{S}_{\mathrm{D}}}{\mathfrak{I}}
$$

Wenn $\mathrm{n}=$ Io gewählt wird, wie es in Fig. 2 der. Fall ist, so gilt nach Gl. 2o), 2I) und 23) für die Konstruktion eines Porzellanisolatorkörpers:

$$
\left.\mathrm{S}_{\mathrm{U}}=5 \mathrm{~S}_{\mathrm{D}} \cdot . \cdot . \cdot . \cdot . \cdot . \cdot 25\right)
$$

1) Ich beabsichtige, hauptsächlich die Form- und nicht die Materialfrage der Isolatoren zu erledigen. Als Material für den Isolatorkörper soll deshalb für die Berechnungen in dieser Schrift das manchen andern Stoffen punkto Qualität nachstehende, seiner leichten Formbarkeit und Billigkeit wegen aber sonst viel gebräuchliche Porzellan vorgezogen werden. 
und also:

$$
\left.\Delta t=\frac{S_{D}}{2} \ldots \ldots . . . . . .26\right)
$$

Mit dem so gefundenen Werte $\Delta \mathrm{t}$ denken wir uns im Vakuumfelde (Fig. 2) aus beiden k.I-Scharen beliebig viele Kurven, die gleichmäßig über das Feld verteilt sein mögen, konstruiert. Alle diese Kurven besitzen die gleiche Gesamtlänge $S_{U}$ und die gleiche konstante Tangentialfeldstärke $\mathfrak{T}$; sie sind einander also in elektrischer Hinsicht vollständig äquivalent. Wir können darunter irgendeine Kurve zur Festlegung der äußern Begrenzung des Isolatorkörpers wählen.

Behufs Raumersparnis in Kästen von Transformatoren, Ölschaltern usw. ist ein Isolator von möglichst kleinen Dimensionen von Vorteil. Der Verfasser hat sich deshalb für eine $k \cdot \mathfrak{T}$-Kurve entschieden, wie sie Fig. I zeigt. Dieșe Kurve entfernt sich an der Niederspannungsfassung $\mathrm{N}$ beginnend vom Mittelteile $\mathrm{M}$ des Isolators, kehrt dann aber im Verlaufe zur Hochspannungsfassung hin wieder gegen diesen zurück. Die Wahl wurde noch durch den glücklichen Umstand begünstigt, daß diese Kurve die Hochspannungsleitung nicht an einer ihrer engen, sondern an ihrer weitesten Stelle, nämlich an der äußern Begrenzung des Spindelkopfes $S$ trifft.

Der neue Isolator wird deshalb an der Hochspannungsfassung bei weitem weniger beansprucht als die gewöhnlichen Durchführungsisolatoren mit ihren engen Fassungen.

Unter anderem wurde vom Verfasser auch Wert darauf gelegt, den. Isolatorkörper im generellen tangential an die Leiter heranzuführen, wodurch die Tangentialfeldstärke längs des Isolatormantels an den Fassungsstellen bis auf jeden gewünschten Kleinheitsgrad gebracht werden kann. Dariber gibt auch das Beispiel des vorigen Abschnittes näheren Aufschluß. An dem dort vorkommenden linearen Leiter ist die Feldstärke sogar unendlich groß und die k.T-Kurven laufen deshalb genau tangential in ihn ein.

\section{Hilfssätze aus der Potentialtheorie.}

Wir betrachten das elektrostatische Feld eines Leitersystemes $A$, das aus den beliebig gestalteten, in fester Konfiguration zueinander stehenden Konduktoren $K_{1}, K_{2}, K_{3} \ldots K_{n}$ bestehen möge, im freien Äther. Jeden Konduktor denken wir uns durch eine kapazitätslose Leitung mit einer unendlichfernen Elektrizitätsquelle von konstantem Potentiale verbunden.

Die Potentialfunktion $\varphi_{\mathrm{I}}$ unseres Systemes besitzt dann an den einzeinen Konduktoren Leiterpotentiale $\Phi_{1}, \Phi_{2}, \Phi_{3} \ldots \Phi_{\mathrm{n}}$, die durch elektrostatische Influenz nicht geändert werden können.

Nun werde vom Unendlichen her ein neuer Konduktor K mit der Gesamtmasse Null in unser Feld eingeführt. Die nunmehr existierende Potentialfunktion $\varphi_{\text {II }}$ besitzt dann am eingeführten Konduktor $\mathrm{K}$ einen gewissen konstanten Randwert $\mathrm{C}$, den wir abgrenzen wollen.

$\mathrm{Zu}$ diesem $Z$ wecke betrachten wir die Differenz:

$$
\left.\psi=\varphi_{\mathrm{I}}-\varphi_{\mathrm{II}} \ldots . . . . . . . .27\right)
$$

Auf Grund der Eigenschaften von $\varphi_{\mathrm{I}}$ und $\varphi_{\mathrm{II}}$ ist $\psi$ eine Potentialfunktion mit den Randwerten o an allen Konduktoren des Systemes $A$ und rom Betrage

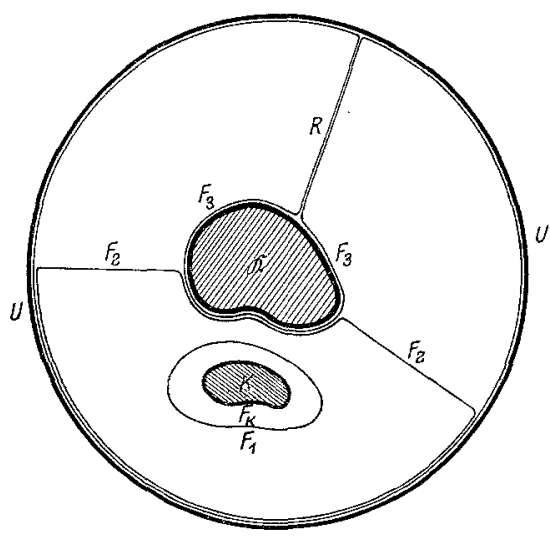

Fig. 5 . $\Psi=\Phi-\mathrm{C} \ldots \ldots . .28$ am Konduktor K, wobei $\Phi$ die Potentialverteilung des ursprïnglichen Feldes $\varphi_{1}$ an $\mathrm{K}$ bedeutet.

Für alle Untersuchungen betreffend der Funktion $\psi$ repräsentiert das Leitersystem $A$ einen einzigen geerdeten Leiter $\mathfrak{\Re}$; denn wir können uns dafür die einzelnen Konduktoren durch Drähte verbunden denken.

Da die Gesamtmasse auf $\mathbf{K}$ gleich Null ist, verschwindet das Oberflächenintegral der Normalkomponente der elektrischen Feldstärke $\mathfrak{C}$ äber jeder Fläche, die $K$ gan $z$ umschliefit, Es ist also:

$$
\left.\mathrm{J}=\int_{(\mathrm{F})} \mathbb{E}_{\mathrm{n}} \mathrm{df}=\mathrm{o} \ldots \ldots .29\right)
$$

Dies gilt $z$. B. für die Flächen $F_{1}, F_{2}$ und $F_{3}$ der Fig. 5. U bedeutet in jener Abbildung die unendlichferne Kugel. $F_{3}$ umschliefit also den ganzen Raum zwischen $\Re$ und $\mathrm{U}$. 
Als Verbindung der Oberflächen von $\Re$ und $U$ ist der unendlich dïnne, röhrenförmige Mantel $\mathrm{R}$ ubrig geblieben. Über diesen verschwindet, wie leicht einzusehen ist, der Integralanteil von $J$. Die restierende Integrationsfläche $\mathrm{F}_{\Re}+\mathrm{U}$ wollen wir durch Einführung der Bezeichnung $F_{\Re, U}$ zusammenfassen. Da sowohl an $F_{\Re}$, wie auch an $U \mathscr{E}_{n}$ mit $\mathscr{E}_{\mathfrak{E}}$ selber identisch ist, kann man für G1. 29) setzen:

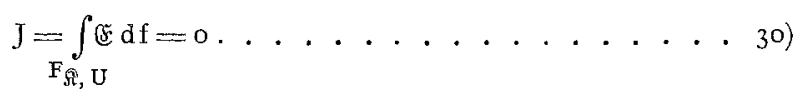

Da $\left(\mathscr{C}\right.$ an $F_{\Re}$ nicht Null ist, folgt aus G1. 3o) mit Notwendigkeit, daß̧ $\left(\mathbb{S}\right.$ an $F_{\Re, U} \mathrm{U}$ teils positiv und teils negativ sein muf. Da aber das Potential $\psi$ auf $\mathbf{F}_{\Re, U}$ allenthalben Null ist, gilt der Satz:

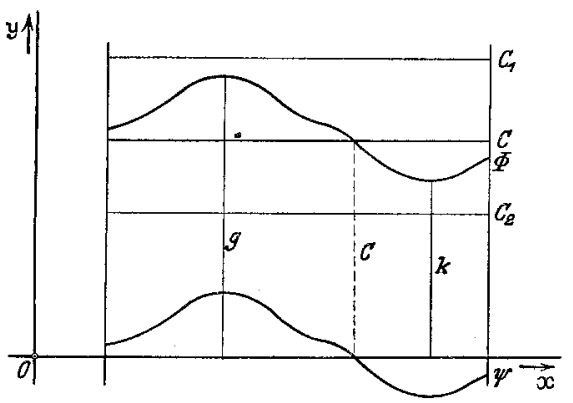

Fig. 6.

Satz I. Das Potential irgendwelcher Massen mit der Summe Null, die auferhalb eines geerdeten Konduktors beliebig verteilt sind, ist teils positiv und teils negativ.

Nach einem bekannten Satze aus der Potentialtheorie ${ }^{1}$ ) liegen die Extremalwerte eines Potentiales am Rande des Regularitätsgebietes, wofür $F_{\Re, U}$ und die Oberfläche von $\mathrm{K}$ in Betracht kommen ${ }^{1}$ ). Auf Grund von Satz I gibt es aber mindestens einen positiven und einen negativen Extremalwert und diese können unmöglich auf $\mathrm{F}_{\Re, U} \mathrm{U}$ liegen, weil dort $\psi$ überall verschwindet.

Die Konsequenz ist: Die positiven und die negativen Extremalwerte des Potentiales $\psi$ liegen alle an $\mathrm{K}$.

Daraus resultiert aber des stetigen Verlaufes einer Potentialfunktion wegen für die Funktion $\Psi[G 1,281]$ :

Die Randfunktion $\Psi$ ist teils positiv und teils negativ.

Nach G1, 28) ist:

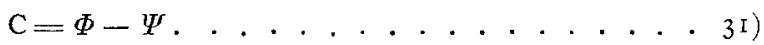

Bezeichnet $\mathrm{g}$ den gröfíten und $\mathrm{k}$ den kleinsten Wert von $\Phi$, so erkennt man an Hand von Fig. 6, dafi für jeden Fall, wo $\Psi$ teilweise positiv und teilweise negativ ist, die Ungleichung bestehen muf:

$$
\mathrm{k}<\mathrm{C}<\mathrm{g}, \ldots . . . . . . . . .32)
$$

was in Worten ausgedruickt lautet:

Satz II. Bringt man irgendeinen ungeladenen Konduktor $K$ in das statische Feld eines Systems von Leitern $(\Lambda)$, deren Potentiale durch äufiere Elektrizitätsquellen konstant gehalten werden, so liegt das entstehende konstante Leiterpotential $\mathrm{C}$ von $\mathrm{K} z$ wischen dem maximalen und minimalen Potentialwerte des ursprünglichen Feldes an der Oberfläche von K.

Zusatz: Das Leiterpotential $C$ ist also ein gewisser Mittelwert der Randwerte $\Phi$ des ursprünglichen Feldes an der Oberfläche von $\mathrm{K}$.

\section{Potentialregulatoren.}

Aus dem Konduktor $\mathrm{K}$ des vorigen Abschnittes kann man durch einen Grenzübergang eine leitende Fläche $\lambda$ erhalten. Auf einer solchen Fläche $\lambda$ ist dann nirgends

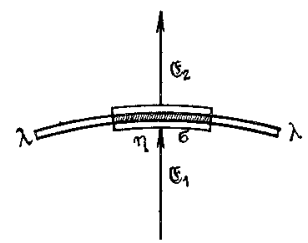

Fig. 7 . elektrische Dichte vorhanden, wenn sie sich vollständig mit einer Niveaufläche des ursprünglichen Feldes $\varphi_{\mathrm{I}}$ deckt. In jedem andern Falle kommen darauf positive und negative Belegungen $\eta$ vor.

Wenden wir den Gaußschen Integralsatz auf ein über dem Flächenstück $\sigma$ der Fläche $\lambda$ errichtetes Prisma von sehr kleiner Höhe an (vgl. Fig. 7), so erhalten wir:

$$
\int_{\sigma} \dot{B}_{2} \mathrm{~d} \lambda-\int_{\sigma} \dot{B}_{1} \mathrm{~d} \lambda=4 \pi \int_{\sigma} \eta \mathrm{d} \lambda
$$

oder also, wenn $\mathrm{N}_{1}$ den eintretenden und $\mathrm{N}_{2}$ den austretenden Fluß und $\mathrm{e}_{\sigma}$ die Elektrizitätsmenge auf $\sigma$ bedeuten:

$$
\mathrm{N}_{2}-\mathrm{N}_{1}=4 \pi \mathrm{e}_{\sigma} \text {. . . . . . . . . . . . . 34) }
$$

1) Vgl. C. Neumann, Untersuchungen ïber das logarithmische und Newtonsche Potential, $\oint$ I I und $\S$ I 2 . 
Formel 34) besagt uns, daß die auf die Fläche $\lambda$ auftreffenden Flußröhren daselbst nicht eine stetige Fortsetzung finden. Erstreckt man die Integrationen der Formel 33) hingegen auf die ganze Fläche $\lambda$, so erkennt man nach Formel 34), daß der totale in $\lambda$ eintretende Fluß gleich dem totalen aus $\lambda$ austretenden Flusse sein muß; denn es ist:

$$
\mathrm{e}_{\lambda}=\text { o. . . . . . . . . . . . . 35) }
$$

Solche leitende Flächen sind z. B. Bleche odèr sonstige Leiterbelege, die als Potentialregulatoren, d. h. als Einrichtungen zur Ausgleichung von lokalen Spannungsänderungen in das Feld eingeführt werden. Stehen diese Bleche längs Niveauflächen des ursprünglichen Feldes $\varphi_{\mathrm{I}}$, so werden darauf nach den vorhergehenden Betrachtungen keine elektrischen Belegungen induziert. Die Flußröhren finden durch dieselben ihre stetige Fortsetzung. Gibt man den Blechen hingegen ganz willkürliche Formen, so werden darauf bestimmte elektrische Verteilungen entstehen, und das Feld wird so deformiert, daß die Eintritts- und Austrittsstellen der Flußröhren an den Blechen gegeneinander verschoben sind.

Der Isolator (vgl. Fig. 8 und 9) ist mit einem solchen Bleche ausgestattet. Der konstante Potentialwert $C$, der sich nach dem Zusatze des vorigen Abschnittes darauf einstellt, ist ein Mittelwert aus den Potentialwerten des ursprünglichen Feldes $\varphi_{\mathbf{I}}$ längs dieses Bleches. Solange das durch das Blech hervorgerufene Superpositionsfeld gegenüber dem Feld $\varphi_{I}$ in den Hintergrund tritt, kann mit hinreichender Geuauigkeit dieser Mittelwert durch das arithmetische Mittel aus dem maximalen und minimalen Randwerte des Potentiales $\varphi_{\mathrm{I}}$ am Bleche ersetzt werden.

Das verwendete Blech ist nicht längs einer Niveaufläche des Vakuumfeldes gelegt, sondern so geformt, daß die Feldstärke in der Durchführungsstelle auch nach Einführung des Isolatorkörpers nur unwesentlich geändert wurde. Im Bilde (Fig. 8) läßt sich am Verlauf der $\mathfrak{D}$-Linien, welche am Bleche B Unterbrechungen erfahren, sehr schön erkennen, daß dieses induzierte Ladungen enthält, durch welche bei der ausprobierten Leiterform die gewünschte Felddeformation erreicht ist. Zur Erhöhung der Sicherheit wird es manchmal vorteilhaft sein, statt nur ein Blech, deren mehrere zu verwenden. Der Hauptzweck dieser Bleche besteht in der Ausgleichung von Störungen, die in der Potentialverteilung auftreten können. Wenn die Potentialdifferenz zwischen Hochund Niederspannung gleichbleibt, sind sie immer auf irgendeinen Teil des Isolators lokalisiert und können durch diese mit andern Feldpartien in Verbindung stehenden Bleche teilweise ausgeglichen werden. Von großem Vorteile sind sie daher für die Aufrechterhaltung der Spannungsverteilung in der Durchführungsstelle und besonders längs des Isolatorkörpers.

\section{Das D-Linienbild.}

Die Konstruktion des D-Linienbildes (Fig. 8) wurde nach Annahme des Isolatorkörpers unter der. Voraussetzung einer Dielektrizitätskonstanten $\varepsilon=5$ für das verwendete Isolationsmaterial vorgenommen. Für die Flußröhren wurde die gleiche Verschiebungsmenge gewählt, wie im Vakuumfeld (Fig. 2).

Das $\mathfrak{D}$-Linienbild zeigt als Hauptcharakteristikum für den neuen Durchführungsisolator, daß die meisten D-Linien den Isolatorkörper zweimal und nicht nur einmal, wie dies bei gewöhnlichen Isolatoren der Fall ist, durchsetzen. Die Anzahl der Verschiebungsröhren ișt hier größer als die Anzahl der Flußröhren im Vakuumfelde. Die Niveaulinien sind hier im Hohlraum des Isolators deshalb etwas gedrängter als an der entsprechenden Stelle dort. Immerhin ist die Äquidistanz derselben im Luftraum des Isolators fast vollständig erhalten, und sie treten auch so durch den Isolatorkörper hindurch, daß die Tangentialfeldstärke an demselben nicht stark variiert. Die vom Ver- 
fasser in Abschnitt 3 gemachte Annahme der ungefähren Übereinstimmung der diesbezüglichen Eigenschaften im Vakuum- und definitiven Isolatorfelde behält also ihre Berechtigung.

An der Oberfläche des Isolatorkörpers zeigen sich recht beträchtliche Brechungen der D- und Niveaulinien. Bei Verwendung von Isolationsmaterialien für den Isolatorkörper, deren Dielektrizitätskonstante $\varepsilon$ kleiner als bei Porzellan ist, würden diese Brechungen und die Abweichungen zwischen Vakuum- und $\mathfrak{D}$-Linienbild auch geringer ausfallen.

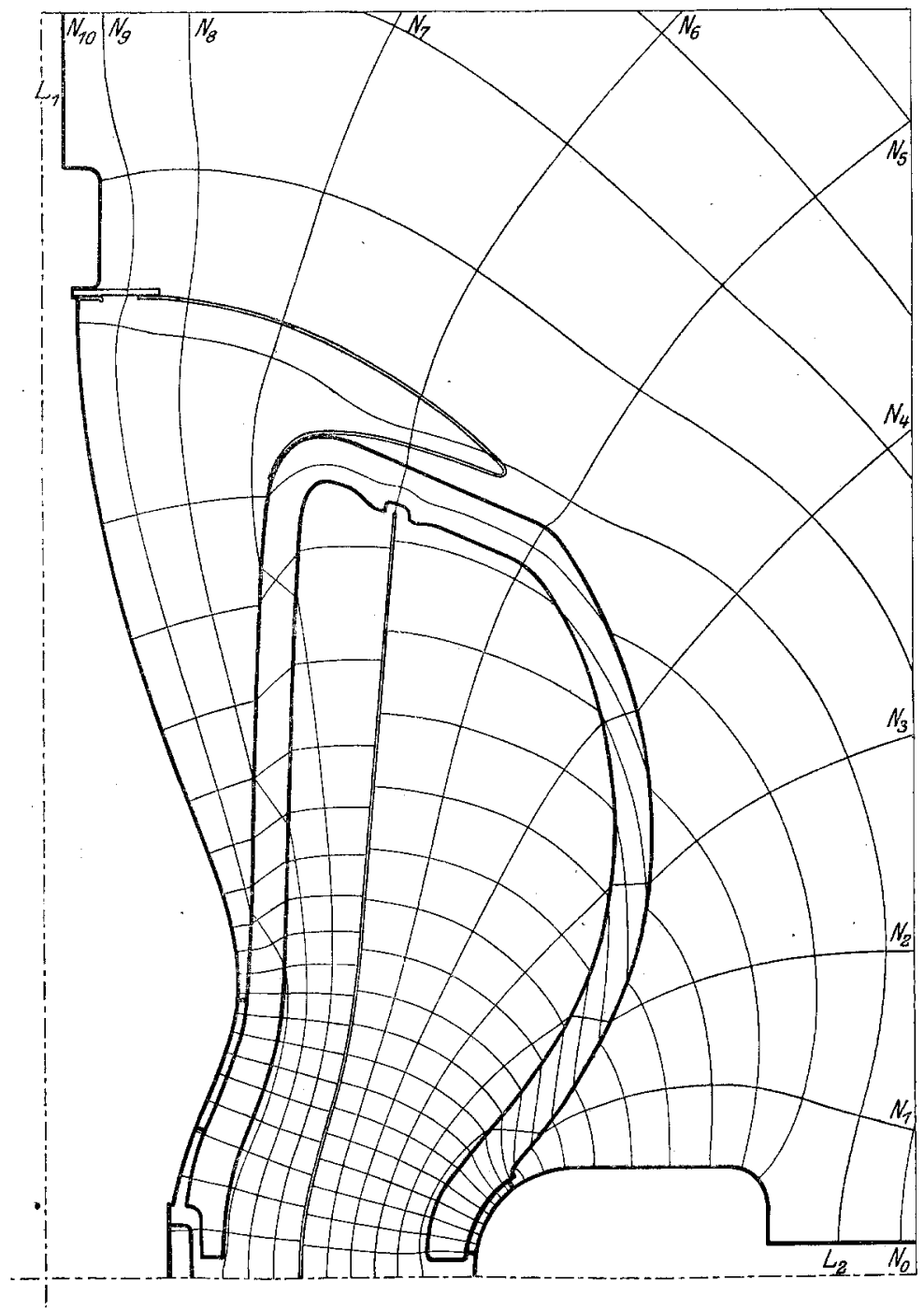

Fig. 8. Meridianschnitt durch das $\mathfrak{D}$-Linienbild des Isolators.

Zur Erhöhung der innern Isolationsfähigkeit kann selbstverständlich auch bei diesem neuen Isolatortypus Druckluft- oder Ölfüllung verwendet werden. Beim Gebrauche von Öl $(\varepsilon=2,2-2,3)$ würde die Brechung an der Innenfläche des Isolatorkörpers auch vermindert und die Potentialverteilung in der Durchführungsstelle derjenigen des Vakuumfeldes ähnlicher. Also würde die Verwendung dieser Füllungen nur zu empfehlen sein. Die diesbezügliche theoretische Untersuchung ist in dieser Schrift übergangen worden. 


\section{Zur Konstruktion des Isolators.}

Der ganze Isolatorkörper besteht aus zwei Hälften, wovon die zweite zu der in den Fig. 8 und 9 gezeichneten in bezug auf die Durchführungsstelle symmetrisch liegt. Die beiden Teile werden durch eine in der Doppelspindel $S_{1} S_{2}$ (siehe Fig. I und 9) zwischen den Hochspannungsfassungen verlaufende Schraube tangential gegen die Niederspannungsfassung gepreßt. Die Zweiteiligkeit des Isolatorkörpers ermöglicht die Verwendung des Isolators bei Wänden von verschiedener Dicke. Er eignet sich auch für sehr dicke Wände. Zur Erlangung einer angenähert konstanten Feldstärke in der Durchführungsstelle ist es dann doch zweckmäßig, die Einschnürung der Doppelspindel wenn auch nur unwesentlich zu vergrößern.

Der Isolatorkörper wird am äußeren Teile mechanisch auf Druck und am innern Teile (am Trichter) auf Zug beansprucht. Die Dicke seiner Wandung ist mit Rücksicht darauf gewählt worden. Für die rein elektrische Beanspruchung wäre ein dünnerer Isolatormantel vorteilhafter.

Der Isolator hat nicht die längliche Form der gewöhnlichen Durchführungsisolatoren, sondern wird gegen außen beinahe kugelförmig begrenzt. Der Gleitfunkenweg ist nichtsdestoweniger kürzer, denn es gehört dazu auch der Weg längs des Trichters, in dessen Tiefe die Hochspannungsfassung sitzt.

Der Isolator ist in der Schrift rillenlos konstruiert; doch sei hier bemerkt, daß die Anbringung von Rillen keine Schwierigkeiten mit sich bringt. Durch die Rillen würde der Gleitfunkenweg noch wesentlich vergrößert und

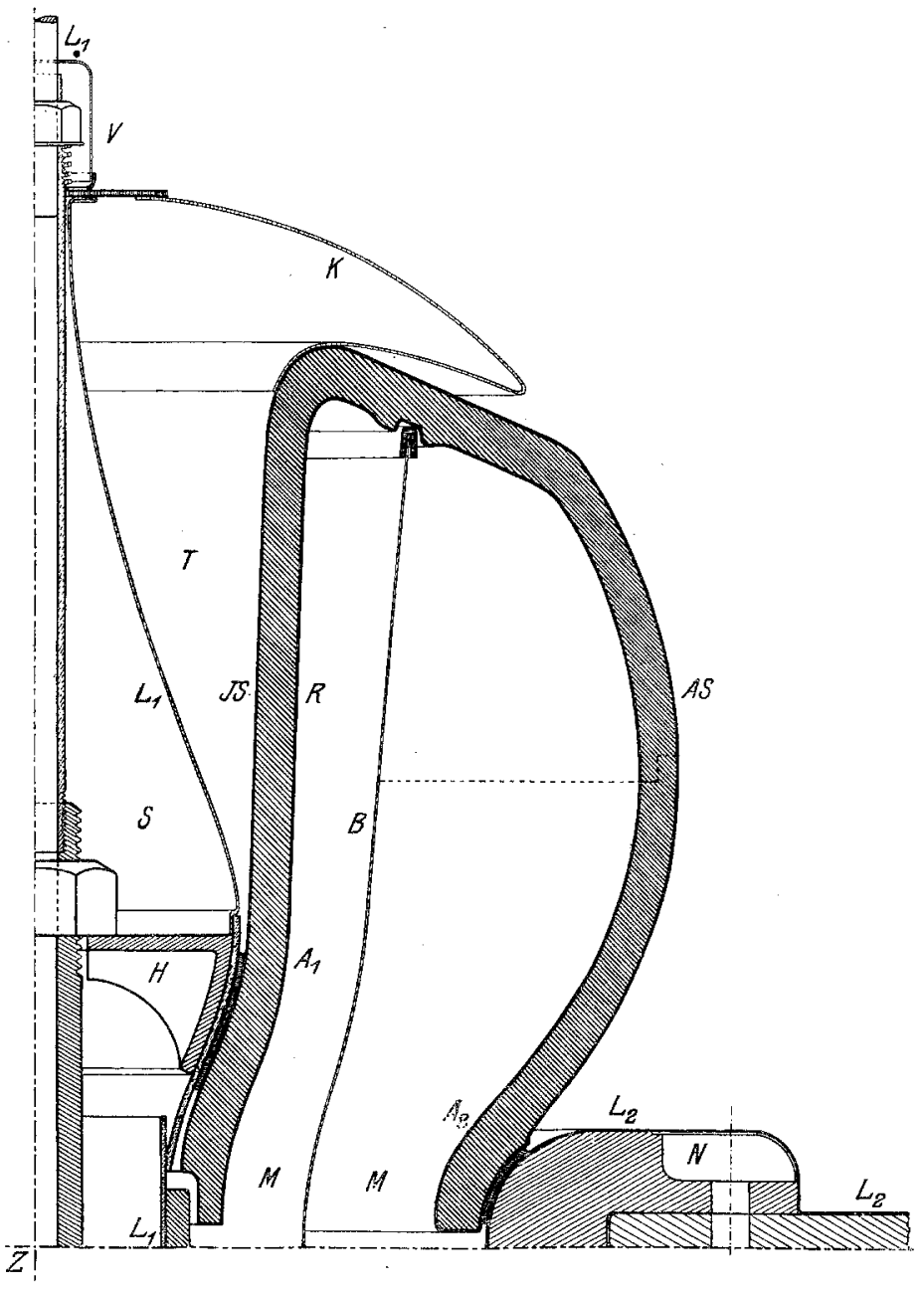

Fig. 9. Durchführungsisolator. im Falle stärkerer Ionisationen würde das Auftreten von Überschlagsfunken erschwert. Die Rillen würden hier in irgendwelchen wulstartigen Ausbuchtungen am Isolatormantel bestehen.

Um die Hochspannungsleitung ungehindert an den Isolator heranführen zu können, befindet sich der eigentliche Leitungsanschluß nicht im Zentrum der Hochspannungsfassung, sondern. im Verschlusse $V$ eines röhrenförmigen Ansatzes.

Zum Schutze der Trichter $\mathrm{T}$ gegen Verstaubung und das Eindringen sonstiger Fremdkörper können Schutzkappen $\mathrm{K}$, wie dies in Abb. 9 erkenntlich ist, verwendet werden. An jeder solchen Schutzkappe ist die Tangentialfeldstärke in der Nähe der Hochspannungsleitung (vgl. Fig. 8) größer als längs des Isolatorkörpers. Es empfiehlt sich deshalb, dazu ein für das Entstehen von Gleitfunken noch ungünstigeres Material 
als am Isolatorkörper zu verwenden. Es eignen sich dafür selbst Stoffe, die keine große mechanische Festigkeit besitzen, wie imprägniertes Papier, Gummi- und Mikapräparate. Eine zweckmäßige Abdichtung mit der Hochspannungsleitung erzielt man mit einer Glimmerplatte (siehe Fig. 8). Eine etwas einfachere, in elektrischer Hinsicht vielleicht etwas günstigere Modifikation der Schutzkappen $\mathrm{K}$ würde man erhalten durch Weglassen des vom Falze zurücklaufenden und auf dem Isolatorkörper aufliegenden Teiles der Kappe. Bei dieser Änderung wäre aber der Trichter $\mathrm{T}$ nach außen nicht mehr vollständig abgeschlossen.

Der durch diese Abhandlung eingeführte Durchführungsisolator besitzt somit, nochmals kurz zusammengefaßt, folgende charakteristișche Eigenschaften:

Die Feldstärken an der Durchführungsstelle sowie längs des Isolatorkörpers sind praktisch konstant. Deshalb besitzt der Isolator eine maximale Widerstandsfähigkeit gegen Durchschlag und Überschlag bei stark reduzierten Dimensionen in radialer und axialer Richtung. Bei gleichen Sicherheitskoeffizienten verhalten sich die Längen dieses Isolators und der gewöhnlichen rund wie I : 2 und die Durchmesser der Niederspannungsfassungen wie $I:{ }^{4} / 3$. Außerdem läßt das tangentiale Anlegen des Isolatorkörpers an die Leiter eine minimale Feldstärke an den Fassungsstellen zu, und die „Spannungsregulatoren" machen das Auftreten von lokalen störenden Spannungsänderungen im Isolatorfeld zum großen Teile unmöglich.

\section{Gibt es geschlossene elektrische Kraftlinien?}

Von

\section{G. Benischke.}

I. Aus der Maxwellschen Hypothese über das elektromagnetische Feld hat man die Folgerung gezogen, daß die elektrischen Kraftlinien eines Wechselstromes geschlossene Linien sind, die parallel zum Leitungsdraht verlaufen. Diese Auffassung wird öfter ausgesprochen, aber nicht zeichnerisch dargestellt, während die elektrischen Kraftlinien ruhender Ladungen oder die magnetischen Kraftlinien elektrischer Ströme sehr häufig aufgezeichnet werden. Man scheint doch noch davor zurückzuschrecken, die angeblichen geschlossenen elektrischen Kraftlinien so bestimmt darzustellen, wie es eben durch eine Zeichnung geschieht. Zum ersten Male finde ich solche in ihrem ganzen Verlauf dargestellt in dem Artikel „Zur Definition der induzierten elektromotorischen Kraft" von Herrn Rogowski in dieser Zeitschrift 4. Bd. S. 56, I9I5. Dort sind in Fig. 2 die elektrischen Kraftlinien einer kreisförmigen Sekundärspule eines Transformators mit zylindrischem Eisenkern als konzentrische Kreise zu dieser Spule gezeichnet. Diese Darstellung wird dort die „heutige Vorstellung“ genannt, während in Fig. I die „alte Vorstellung" gezeichnet ist, die sich von Fig. 2 dadurch unterscheidet, daß im Luftraum keinerlei elektrische Kraftlinien vorhanden sind.

Diese Frage ist von Wichtigkeit nicht nur für die Theorie, sondern auch für die Hochspannungstechnik.

2. Zunächst möchte ich betonen, daß die ,alte" Vorstellung, wie ich sie kenne, nicht darin besteht, daß es im Raum außerhalb des Leiters keine elektrischen Kräfte gibt, sondern darin, daß es elektrische Kraftlinien gibt, die Anfang und Ende haben. In diesem Falle verlaufen sie zum größten Teil zwischen Spule und Eisenkern, zum Teil aber auch zwischen Spule und anderen benachbarten Leitern oder der Erde.

Daß die elektrischen Kraftlinien statischer Ladungen keine geschlossenen Linien sind, sondern Anfang und Ende haben, dürfte wohl nicht bezweifelt werden. Sind z. B. zwei Drahtstücke a b und cd (Fig. I) entgegengesetzt geladen, so nehmen die 